\section{Is Yoga possible for elderly care?}

Siukan Law, ${ }^{1}$ Albert Wingnang Leung, ${ }^{2}$ Chuanshan $\mathrm{Xu}^{3}$

${ }^{1}$ Department of Science, School of Science and Technology, The Open University of Hong Kong, Ho Man Tin, Kowloon, Hong Kong; ${ }^{2}$ School of Nursing, Tung Wah College, Ho Man

Tin, Kowloon, Hong Kong; ${ }^{3}$ Key Laboratory of Molecular Target and Clinical Pharmacology, State Key Laboratory of Respiratory Disease, School of Pharmaceutical Sciences \& Fifth Affiliated Hospital, Guangzhou Medical University, Guangzhou, China

\section{Abstract}

Yoga is possible for elderly care as it promotes and balance among mind-brainbody functions through mental, physical, and emotional practices. Growing evidence has shown that yoga practices strengthen muscle, body flexibility, and boost the immune system as well as improve the physical and psychological conditions.

\section{Yoga and elderly care}

Yoga is an aerobic exercise, joined the mind concepts and its body to complete well-being healthy through mental, physical, and emotional practices. This is not strenuous exercise and suitable for persons of all ages. It does not have any guidelines, just only breathe to start doing actions in a relaxed mode. The pathophysiological mechanism of yoga focuses on the integration of top-down and bottom-up forms of self-regulation for facilitating the bidirectional feedback in brain networks. Yoga is a useful technique to enhance and balance among mind-brain-body functions for older people. ${ }^{1}$

Some yoga published journals were reviewed by using library search engines such as SCI/SCIE, PubMed, and Scopus. Growing evidence has shown that yoga practices strengthen muscle, body flexibility, and boost the immune system such as respiratory and cardiovascular functions, also improve the physical and psychological conditions including reducing stress, anxiety, depression, chronic pain, and changing sleep patterns to get a better quality of life (Table 1).

Sarbaz et al. reported yoga practices on muscular strength for preventing multiple sclerosis symptoms. A total of 22 participants were selected which arranged a yoga program three times a week at 90 mins per lesson for 6 months. The surface electromyography signals were significantly increased to double after intervention with yoga practice compared to the control groups. $^{2}$

Gopal et al. discovered the yoga practices on immune responses which resisted the autonomic changes and impairment of cellular immunity. A total of 30 participants were selected and arranged a yoga program at 35 minutes daily for 12 weeks. In the yoga group, the cortical increased and serum IFN- $\gamma$ decreased that indicated yoga was effectively via cerebro-cortico-limbic pathways on the hypothalamus and the anterior pituitary systems to enhance a balance between the sympathetic and parasympathetic limbs in an autonomic nervous system. The cytokine level such as IL-4 was increased by the yoga practices and boosted an immune system. ${ }^{3}$

Loganathan et al. indicated yoga practices improved respiratory and cardiovascular functions as they strengthened the inspiratory and expiratory muscles, regulated breathings to prolong the breathing time. It also significantly lower systolic blood pressure, pulse pressure, and mean arterial pressure for blood circulation to prevent respiratory and cardiovascular diseases. ${ }^{4}$

Cramer et al. identified regular yoga practices associated with increased physical health in chronically diseased patients, especially in improving health status and quality of life in patients with arthritis. The isometric exercises practiced during yoga had been shown to relieve pain and muscle spasms. It increased awareness of muscle tonus and joint position which help to recognize and change the habitual patterns of posture and muscle tension in daily life for treating and preventing chronic pain. ${ }^{5}$

Bankar et al. reported regular yoga exercises at least one hour daily for elderly people could help to achieve good sleep quality that was associated with fewer sleep disturbances. Yoga might down-regulation of 5HT1A (5-hydroxytryptamine 1A) receptors, decrease autonomic over-activity and increase parasympathetic activity with

Table 1. Major benefits of yoga for the elderly.

\section{Strengthen muscle flexibility}

Increase lung capacity

Regulate blood pressure

Reduce chronic pain

Stress relief
Correspondence: Siukan Law, Department of Science, School of Science and Technology, The Open University of Hong Kong, Ho Man Tin, Kowloon, Hong Kong.

E-mail: siukanlaw@hotmail.com

Key words: Yoga; elderly care.

Contributions: the authors contributed equally.

Conflict of interests: the authors declare no potential conflict of interests.

Received for publication: 16 April 2021.

Revision received: 26 May 2021.

Accepted for publication: 25 June 2021.

This work is licensed under a Creative Commons Attribution-NonCommercial 4.0 International License (CC BY-NC 4.0).

${ }^{\circ}$ Copyright: the Author(s), 2021

Licensee PAGEPress, Italy

Geriatric Care 2021; 7:9815

doi:10.4081/gc.2021.9815

reduction of oxygen consumption and metabolic rate of prefrontal cortex cells to prevent disturbing sleep. ${ }^{6}$

The above information demonstrates that yoga is possible for elderly care. Strengthen muscle, body flexibility, boost the immune system as well as improve the physical and psychological conditions through the 8 limbs of yoga practices involving relaxation (shava asana), physical postures (asana), breathing regulation techniques (pranayama), and meditation (dhyana) to promote a healthy body.

\section{References}

1. Gard T, Noggle JJ, Park CL, et al. Potential self-regulatory mechanisms of yoga for psychological health. Front Hum Neurosci 2014;8:770.

2. Sarbaz Y, Beni KN, Hosseininejad A, et al. The effect of yoga practice on muscular strength improvement in patients with multiple sclerosis. Int $J$ Ther Rehabil 2020;27:1-10.

3. Gopal A, Mondal S, Gandhi A, et al. Effect of integrated yoga practices on immune responses in examination stress - A preliminary study. Int J Yoga 2011;4:26-32.

4. Loganathan N, Aruchunan M, Manjunath NK. Effects of yoga for cardiovascular and respiratory functions: a pilot study. Integr Med Res 2019;8:180.

5. Cramer H, Lauche R, Langhorst J, et al. Quality of life and mental health in 
patients with chronic diseases who regularly practice yoga and those who do not: a case-control study. Evid Based Complement Alternat Med 2013;2013: 702914.
6. Bankar MA, Chaudhari SK, Chaudhari KD. Impact of long term Yoga practice on sleep quality and quality of life in the elderly. J Ayurveda Integr Med 2013;4: 28-32. 